\title{
TWO VALUES OF THE CONSTANT G OBTAINED FROM INCLINING-PULL PROJECTION METHOD \\ HU QING-GUI ${ }^{1}$
}

Modern Education Technology Centre, Neijiang Normal University, Neijiang, Szechwan Province, China

\section{ABSTRACT}

The Newtonian gravitational constant is regarded as the most fundamental constant. So far, more than 200 experiments have been done to measure its true value. But large discrepancies in those results appeared. Owing to the weakness of the gravitational interaction and the impossibility of shielding its effects, it is very difficult to find new method to measure $G$. Thus, most previous experiments were based on the torsion pendulum or torsion balance. Here we report the inclining-pull projection method to measure the gravitational constant $G$. The experimental idea is completely different from previous. With different center distances of two balls, we do this experiment two times and gain two values: $G=6.858448 \times 10^{-11} \mathrm{~m}^{3} \mathrm{~kg}^{-1} \mathrm{~S}^{-2}$, with an uncertainty of about $2.5 \% ; G=6.785245 \times 10^{-11} \mathrm{~m}^{3} \mathrm{~kg}^{-1} \mathrm{~S}^{-2}$, with the uncertainty of about $2.3 \%$. It implies it is possible for $G$ not to be a constant.

KEYWORDS: Gravitational Constant $G$, Torsion Balance, Precision, Displacement, PACS Serial Number-92.60.Dj

The gravitational constant, $G$, is very important because of its key role in many fields such as gravitation, geophysics cosmology, particle physics and so on (Cavendish, 1798) (Schlamminger, 2014). In 1798, British physicist Cavendish used the torsion balance to gain its value $G=(6.67 \pm 0.07) \times 10^{-11} \mathrm{~m}^{3} \cdot \mathrm{kg}^{-1} \cdot \mathrm{s}^{-2}$. Since then, more than two hundred experiments have been done to measure its actual value. However, the accuracy has not been improved much. In 2016, the Committee on Data for Science and Technology published an updated recommended value (CODATA-2014), $6.67408 \quad$ (31) $\times 10^{-11} \mathrm{~m}^{3} \cdot \mathrm{kg}^{-1} \cdot \mathrm{s}^{-2}$ with a relative uncertainty of 47 parts per million (p.p.m.) (Gibney, 2014) (Mohr et al., 2016) (Gillies, 1987) (Quinn, 2014). Compared with other important fundamental constants, its uncertainty is still very high. In the CODATA-2014 adjustment, 14 values gained in the past several decades are considered to be the most accurate values. However, there is a gap of 550 p.p.m. between the largest and the smallest. The large discrepancies have made it difficult for us to know its exact value (Luo et al., 2001) (Rothleitner and Schlamminger, 2017) (Li et al., 2016) (Gundlach and Merkowitz, 2000).

Gravity acts everywhere, but its interaction is very weak, it is hard to detect (Liu et al., 2008) (Newman and
Bantel, 1999). This leads to the notable difficulty for us to find new method to measure $G$. Thus, most previous experiments were based on the torsion pendulum or torsion balance originated from Cavendish experiment in 1798. Here we report the inclining-pull projection method to measure the gravitational constant $G$. The experimental scheme is completely different from previous. What's more, the new experiment can determine $G$ between big objects.

In fact, the author had designed this experimental scheme ten years ago. It was published on Journal of Hebei University of Science and Technology in 2008 (Qing-gui, 2008). All the time, he wants to carry out it. However, due to the limitation of funds and experimental conditions, he was not able to do so. After a decade of efforts, last month, the situation changed. The experiment was completed two times successfully to gain two values of $G$. The different values imply it is possible for $G$ not to be a constant.

\section{THE EXPERIMENTAL SCHEME}

As shown in Figure 1, a small object $A$ is hanged by a long thread (about 60 meters). Object $B$ is placed to attract A. The light source is placed to irradiate the small object. The scale screen is placed to receive the shadow of 


\section{QING-GUI: TWO VALUES OF THE CONSTANT G OBTAINED FROM INCLINING-PULL PROJECTION METHOD}

the small object. To avoid the influence of wind, the thread passes through a pipe.

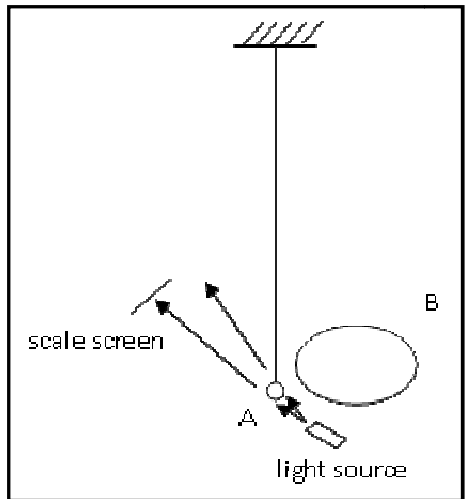

Figure 1: The experiment arrangement. A, suspended object; B, attraction object

In the experiment, at first, we do not put the big object near the small one. We register the shadow position on the scaled screen. Later, we place the big object near the small one to attract it. We register the shadow position again. By comparing the shift value of the shadow, we could determine the gravitational constant $G$.

As shown in Figure 2, when the small ball is attracted by the big one, it is given three forces.

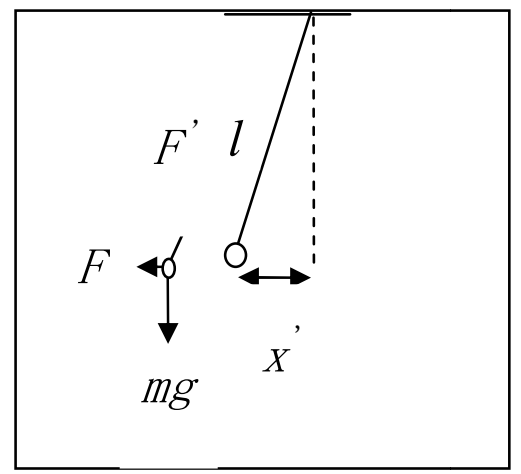

Figure 2: The Force diagram of sphere A. ( $m g$, gravity of sphere $A ; F$, attractive force; $F$, pulling force of the thread; $l$, length of thread; $X$, deviation length of sphere A)
If the gravity of the thread is not taken into account , we can gain the following equation ( $l$ is much longer than $\left.X^{\prime}\left(l>>X^{\prime}\right)\right)$.

$$
F=m g \frac{x^{\prime}}{1}
$$

Where $F$ is the universal gravitation given to ball $\mathrm{A} ; m g$ is the gravity of the ball $\mathrm{A} ; l$ is the length of the thread; $X$ is the displacement of the ball A.

When the gravity of the thread is taken into account,

$$
F=m g \frac{x^{\prime}}{1}+m^{\prime} g \frac{x^{\prime}}{1}
$$

Where $m$ ' is the mass of the thread itself.

On the other hand, according to Newton's law of universal gravitation (Luo et al., 1998) (Yang et al., 2009)

$$
F=G \frac{m M}{r^{2}}
$$

Then, with formula (2) and (3), we can gain the following equation.

$G \frac{m M}{r^{2}}=m g \frac{x^{\prime}}{1}+m^{\prime} g \frac{x^{\prime}}{1}$

It can be rewritten as,

$$
\begin{aligned}
G= & \frac{r^{2}}{m M}\left(m g \frac{X^{\prime}}{1}+m^{\prime} g \frac{X^{\prime}}{1}\right) \\
= & \frac{r^{2} g X^{\prime}}{l M}\left(1+\frac{m^{\prime}}{m}\right)
\end{aligned}
$$


Where $m$ and $M$ are the masses of the ball A and $\mathrm{B}, m^{\prime}$ is the mass of the thread itself, $r$ is the distance between the centers of ball A and B.

In the experiment, we measure the displacement of the shadow to gain shift $X$.

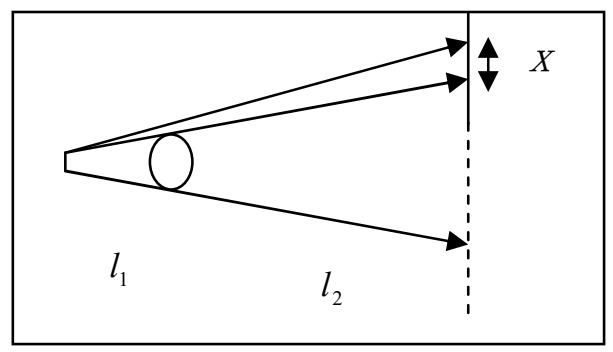

Figure 3: The projection of the suspended ball

As shown in Fig.3, the shape of the light source is not an ideal point. Because the shift of the shadow is very small, we can gain the following equation.

$x^{\prime}=\frac{l_{1}}{l_{1}+l_{2}} X$

Where $X^{\prime}$ and $X$ are the displacements of the ball and its shadow, $l_{1}$ is the distance from light source to ball, $l_{2}$ the distance from ball to receiving screen.

For the experiment, how to measure the displacements of the shadow $(X)$ is the key problem.

\section{THE EXPERIMENT PROCESS}

Please see Figure 1, the experiment includes the following steps.

First step, To suspend sphere A by a long thread. We choose a tall building under construction to do the experiment, where there are ready-made drainage pipe so as to save the cost of laying new pipes. We let the thread pass through the drainage pipe to avoid the influence of wind. In the experiment, we choose a lead ball as sphere A. its weight is $7.26 \mathrm{~kg}$, radius is $6 \mathrm{~cm}$. we choose a big iron ball as sphere B. its weight is $397 \mathrm{~kg}$, radius is $23 \mathrm{~cm}$. Several nylon wires are used to bind the sphere $\mathrm{A}$ so as to suspend it by the long thread. For the thread, it is a long and thin steel wire. Its diameter is $1.2 \mathrm{~mm}$. Its weight is $0.51 \mathrm{~kg}$. Its length is about $65 \mathrm{~m}$. The thin steel wire has the following properties: (a) It can carry a high load, (b) Its length distortion is low. (c) It's stability is strong.

Second step, To install light source. We choose high power LED as the light source, whose model is CY3834D30-20S32P-SYC 300W, product of Shenzhen Chongyang Electric Technology Co., Ltd. It has the following properties: (a) Luminous surface is small, circular luminous surface, diameter $\phi 35 \mathrm{~mm}$. (b) Its optical density is strong. Luminous flux $\varphi$ is up to 30000 lux. (c) The range of light is far. Projective distance is up to $4000 \mathrm{~m}$.

Third step, Installing scaled receiving screen and the illuminometer. To avoid the influence of the ambient light, around and top of screen, we place some boards. Besides, we choose moonless nights to do the experiment. The screen's minimum distinction degree is $1 \mathrm{~mm}$. The probe of a high precision illuminometer is set up on the screen. The position of illuminometer's probe can be adjusted. In the experiment, the shadow on the screen is not clear. There is a bright-dark transition zone. After measurement is finished at one position, the probe moves 1 $\mathrm{mm}$ forward. In this way, we can obtain the different light intensities corresponding to different positions. Up to now, the optical measurement has been very mature. There are many products for us to choose. We select TES-1336 illuminometer, the product of TES Electrical Electronic Corp., Taiwan. Its resolution is 0.01 Lux. Its measuring range is from $0 \sim 20000$ lux. The sampling extraction frequency is 2.5 times / sec.

In the course of the experiment, when we measure the length of thread, new problem occurs. The suspended lead ball keeps swinging slightly. To reduce the influence of wind on the ball, around and top of the suspended lead ball, we place several pieces of boards. In order to get more accurate results, after the lead ball is suspended 24 hours, we begin to measure the length of thread. At this time, 
quiver of the ball becomes slighter. For the question of what causes the quiver, we didn't find the exact reason for the time being. Maybe it's caused by the earth shaking. We measure the length of thread once every 2 minutes. Several measurement values are obtained. We take the average value, which is $63.17 \mathrm{~m}$. Later, we measure the distance between the center of two balls $(r)$. Also, we measure it several times and the average is taken, which is $0.37 \mathrm{~m}$. Afterwards, we measure the distance from light source to lead ball $\left(l_{1}\right)$ and distance from lead ball to receiving screen $\left(l_{2}\right)$, the values are $0.21 \mathrm{~m}$ and $110.12 \mathrm{~m}$ respectively.
Then, we begin to measure illumination distribution at different positions of the shadow. The measurement process is divided into two parts: (a) The bigger object $\mathrm{B}$ is NOT placed near A; (b) The bigger object $\mathrm{B}$ is placed nearby to attract $\mathrm{A}$. The measurement begins at the center of the bright-dark transition zone of the shadow. One value is taken once a minute, ten values are measured at each position. The average is taken. After the measurement is finished at one position, the probe of the illuminometer moves $1 \mathrm{~mm}$ forward. Altogether, the light intensities of 12 positions are measured. The results are shown in Table 1 and Table 2.

Table 1: The light intensities at different points before the ball is attracted /lux

\begin{tabular}{|c|c|c|c|c|c|c|c|c|c|c|c|}
\hline & 1 & 2 & 3 & 4 & 5 & 6 & 7 & 8 & 9 & 10 & Average \\
\hline 1 & 0.21 & 0.17 & 0.22 & 0.21 & 0.18 & 0.23 & 0.16 & 0.15 & 0.18 & 0.22 & 0.193 \\
\hline 2 & 0.53 & 0.54 & 0.67 & 0.66 & 0.52 & 0.43 & 0.47 & 0.51 & 0.49 & 0.47 & 0.529 \\
\hline 3 & 1.01 & 0.92 & 1.09 & 0.97 & 0.82 & 1.14 & 1.15 & 1.07 & 1.02 & 1.06 & 1.025 \\
\hline 4 & 1.13 & 1.09 & 1.07 & 1.21 & 1.02 & 1.17 & 1.15 & 1.21 & 1.03 & 1.12 & 1.120 \\
\hline 5 & 1.23 & 1.22 & 1.37 & 1.31 & 1.19 & 1.28 & 1.31 & 1.19 & 1.12 & 1.29 & 1.251 \\
\hline 6 & 1.34 & 1.31 & 1.59 & 1.37 & 1.41 & 1.57 & 1.38 & 1.31 & 1.37 & 1.45 & 1.410 \\
\hline 7 & 1.73 & 1.91 & 1.79 & 1.71 & 2.03 & 1.65 & 1.68 & 1.63 & 1.59 & 1.68 & 1.740 \\
\hline 8 & 2.04 & 2.17 & 2.14 & 2.1 & 2.07 & 1.98 & 2.08 & 2.09 & 2.19 & 2.25 & 2.111 \\
\hline 9 & 2.23 & 2.27 & 2.41 & 2.32 & 2.43 & 2.14 & 2.38 & 2.11 & 2.21 & 2.12 & 2.262 \\
\hline 10 & 2.52 & 2.63 & 2.48. & 2.32 & 2.84 & 2.47 & 2.47 & 2.52 & 2.57 & 2.62 & 2.551 \\
\hline 11 & 2.82 & 3.02 & 2.87 & 2.92 & 2.83 & 2.95 & 2.78 & 2.71 & 2.98 & 2.83 & 2.871 \\
\hline 12 & 3.01 & 3.17 & 2.98 & 3.13 & 3.19 & 3.15 & 3.06 & 3.11 & 2.98 & 3.02 & 3.080 \\
\hline
\end{tabular}

Table 2: The light intensities at different points after the ball is attracted /lux

\begin{tabular}{|c|c|c|c|c|c|c|c|c|c|c|c|}
\hline & 1 & 2 & 3 & 4 & 5 & 6 & 7 & 8 & 9 & 10 & Average \\
\hline 1 & 0.22 & 0.28 & 0.32 & 0.31 & 0.32 & 0.33 & 0.26 & 0.25 & 0.31 & 0.32 & 0.292 \\
\hline 2 & 0.63 & 0.51 & 0.69 & 0.56 & 0.52 & 0.63 & 0.57 & 0.71 & 0.61 & 0.68 & 0.611 \\
\hline 3 & 1.13 & 1.1 & 1.09 & 1.27 & 1.02 & 1.04 & 1.12 & 1.07 & 1.12 & 1.08 & 1.104 \\
\hline 4 & 1.23 & 1.21 & 1.29 & 1.31 & 1.32 & 1.24 & 1.41 & 1.27 & 1.27 & 1.28 & 1.283 \\
\hline 5 & 1.53 & 1.51 & 1.49 & 1.5 & 1.67 & 1.54 & 1.49 & 1.59 & 1.57 & 1.56 & 1.545 \\
\hline 6 & 1.64 & 1.71 & 1.69 & 1.71 & 1.63 & 1.58 & 1.68 & 1.57 & 1.67 & 1.82 & 1.670 \\
\hline 7 & 1.93 & 1.81 & 1.89 & 2.01 & 1.83 & 1.95 & 2.18 & 1.85 & 1.89 & 1.82 & 1.916 \\
\hline 8 & 2.24 & 2.31 & 2.21 & 2.2 & 2.13 & 2.15 & 2.18 & 2.15 & 2.09 & 2.22 & 2.188 \\
\hline 9 & 2.43 & 2.32 & 2.51 & 2.62 & 2.53 & 2.65 & 2.48 & 2.51 & 2.41 & 2.52 & 2.498 \\
\hline 10 & 2.72 & 2.72 & 2.72. & 2.82 & 2.73 & 2.95 & 2.68 & 2.61 & 2.71 & 2.82 & 2.751 \\
\hline 11 & 3.12 & 3.02 & 2.99 & 3.02 & 3.13 & 3.05 & 2.88 & 3.01 & 2.87 & 3.01 & 3.010 \\
\hline 12 & 3.22 & 3.27 & 3.29 & 3.12 & 3.29 & 3.25 & 3.16 & 3.18 & 2.97 & 3.21 & 3.196 \\
\hline
\end{tabular}




\section{QING-GUI: TWO VALUES OF THE CONSTANT $G$ OBTAINED FROM INCLINING-PULL PROJECTION METHOD}

From above Tab.1 and 2, the light intensities measured in same point are constantly changing. This may be caused by the quiver of the suspended lead ball. In order to calculate the displacement of the shadow, the average value is taken for every point. At the same time, we calculate the fitted line by least square method. Least square method (also known as least square method) is a mathematical optimization technology. It minimizes the sum of squares of errors and finds the best matching function.

We take Tab.1 for example. To take position data as $X$, take the average illumination values as $Y$.

Table 3: The corresponding relation between $X$ and $Y$

\begin{tabular}{|c|c|c|c|c|c|c|}
\hline$X / \mathrm{mm}$ & 1 & 2 & 3 & 4 & 5 & 6 \\
\hline$Y / \mathrm{lux}$ & 0.193 & 0.529 & 1.025 & 1.120 & 1.251 & 1.410 \\
\hline$X / \mathrm{mm}$ & 7 & 8 & 9 & 10 & 11 & 12 \\
\hline$Y / \mathrm{lux}$ & 1.740 & 2.111 & 2.262 & 2.551 & 2.871 & 3.080 \\
\hline
\end{tabular}

To assume $X$ and $Y$ have a linear relationship, they meet the linear equation:

$$
y=a x+b
$$

We adopt the least square method to find the parameters $a$ and $b$, according to the least square method (16-17),

$a=\frac{1}{n} \sum_{i=1}^{n} y_{i}-b \frac{1}{n} \sum_{i=1}^{n} x_{i}$

$b=\frac{n \sum_{i=1}^{n} x_{i} y_{i}-\sum_{i=1}^{n} x_{i} \sum_{i=1}^{n} y_{i}}{n \sum_{i=1}^{n} x_{i}^{2}-\left(\sum_{i=1}^{n} x_{i}\right)^{2}}$

Based on the values of $X$ and $Y$ in Tab.3, we can figure out.

$$
a=0.252
$$

$b=0.0393$

Then, the fitted linear equation:

$y=0.252 x+0.0393$

The meaning of above equation (6) is that the different coordinate position $(x)$ corresponds to different light intensities $(y)$.
In the same way, we can gain the other fitted linear equation from Tab.2 (after the ball is attracted):

$$
y=0.2559 x+0.175
$$

The fitting lines and equations are shown in Figure 4.

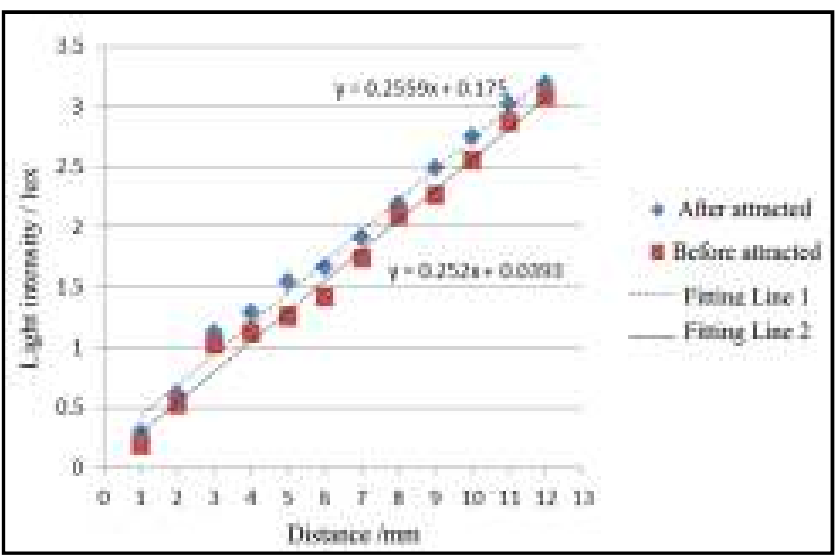

Figure 4: The fitting equations and fitting lines

With equation (6) and (7), we can calculate the moving distance of the shadow like the followings.

First, equation (7) is rewritten as

$$
x=\frac{y-0.175}{0.2559}
$$




\section{QING-GUI: TWO VALUES OF THE CONSTANT G OBTAINED FROM INCLINING-PULL PROJECTION METHOD}

Then, in equation (6), we choose the point $X=$ $1 \mathrm{~mm}$ to calculate the corresponding light intensity on the fitting line 2 .

$$
y=0.252 \times 1+0.0393=0.2913 \operatorname{lux}
$$

For the light intensity value 0.2913 lux, in equation (8), we can calculate the corresponding point $X$ on the fitting line 1.

$$
x=\frac{y-0.175}{0.2559}=\frac{0.2913-0.175}{0.2559}=0.4544744 \mathrm{~mm}
$$

The above equations mean the light intensity at point $X=1 \mathrm{~mm}$ on fitting line 2 (before lead ball is attracted) equals to that at point $X=0.4544744 \mathrm{~mm}$ on fitting line 1 (after the lead ball is attracted). Then, we can speculate the moving distance of the shadow is:

$1 \mathrm{~mm}-0.4544744 \mathrm{~mm}=0.5455256 \mathrm{~mm}$

In the same way, we choose other points $X=2 \mathrm{~mm}$; $3 \mathrm{~mm} ; 4 \mathrm{~mm} \Lambda$ to calculate the moving distance of the shadow, the results are shown in Table 4.

Table 4: The calculated displacement values of the shadow at different points

\begin{tabular}{|c|c|c|c|c|c|c|}
\hline Point & 1 & 2 & 3 & 4 & 5 & 6 \\
\hline shift distance $/ \mathrm{mm}$ & 0.5455256 & 0.5607652 & 0.5760052 & 0.5912452 & 0.6064852 & 0.6217252 \\
\hline Point & 7 & 8 & 9 & 10 & 11 & 12 \\
\hline shift distance $/ \mathrm{mm}$ & 0.6369652 & 0.6522052 & 0.6674452 & 0.6826852 & 0.6979252 & 0.7131652 \\
\hline
\end{tabular}

As shown in Tab.4, at different points, we figure out different shift distance values of the shadow. Then, we take the average as the actual displacement value. Average $\bar{X}$ equals to:

$$
\bar{x}=\frac{1}{12}
$$

$\times(0.5455256+0.5607652+0.5760052+0.5912452+0.606485$

$2+0.6217252+0.6369652+0.6522052+0.6674452+0.682685$

$2+0.6979252+0.7131652) \mathrm{mm}$

Then,

$$
\bar{X}=0.6293452 \mathrm{~mm}
$$

To estimate the credibility of the average value, we adopt type A uncertainty evaluation, the uncertainty $u_{A}$,

$$
u_{A}=\sqrt{\frac{\sum_{i=1}^{n}\left(\bar{x}-x_{i}\right)^{2}}{n(n-1)}}
$$

Where $n$ is the number of statistics times, it is 12 ; $X i$ is statistics value. Then, we can calculate out $u_{A}$,

$$
u_{A}=\sqrt{\frac{\sum_{i=1}^{n}\left(\bar{x}-x_{i}\right)^{2}}{n(n-1)}}=0.015862
$$

Proportion of uncertainty $\delta$,

$$
\delta=\frac{u_{A}}{-} \times 100 \%=\frac{0.015862}{0.6293452} \times 100 \%=2.5 \%
$$

In the following next step, we calculate the actual shift distance of the lead ball. Consider former equation 5 , $X^{\prime}=\frac{l_{1}}{l_{1}+l_{2}} x$, where $I_{1}$ and $I_{2}$ are $0.21 \mathrm{~m}$ and $110.12 \mathrm{~m}$ respectively. The actual shift distance of the ball is 


\section{QING-GUI: TWO VALUES OF THE CONSTANT G OBTAINED FROM INCLINING-PULL PROJECTION METHOD}

$$
\begin{aligned}
x^{\prime}= & \frac{I_{1}}{l_{1}+I_{2}} x=\frac{0.21}{0.21+110.12} \times \bar{x} \\
& =0.00190338 \times 0.6293452 \mathrm{~mm}=1.19788 \times 10^{-3} \mathrm{~mm}=1.19788 \times 10^{-6} \mathrm{~m}
\end{aligned}
$$

Finally, we can calculate the gravitational constant

$G$. Consider former equation 4,

$$
\begin{aligned}
& G= \frac{r^{2}}{m M}\left(m g \frac{X^{\prime}}{1}+m^{\prime} g \frac{X^{\prime}}{l}\right) \\
&=\frac{r^{2} g X}{I M}\left(1+\frac{m^{\prime}}{m}\right) \text { respectively. Then, } G \text { can be counted out by, } \\
& G=\frac{r^{2} g X}{I M}\left(1+\frac{m^{\prime}}{m}\right) \\
& \quad=\frac{0.37^{2} \times 9.8 \times 1.19788 \times 10^{-6}}{63.17 \times 397}\left(1+\frac{0.51}{7.26}\right)=6.858448 \times 10^{-11} \mathrm{~m}^{3} \mathrm{~kg}^{-1} \mathrm{~S}^{-2}
\end{aligned}
$$

$7.26 \mathrm{~kg}, 397 \mathrm{~kg}, 1.19788 \times 10^{-6} \mathrm{~m}, 63.17 \mathrm{~m}$ and $0.51 \mathrm{~kg}$
Thus, we obtain the constant value $G=6.858448 \times 10^{-11} \mathrm{~m}^{3} \mathrm{~kg}^{-1} \mathrm{~S}^{-2}$.

To estimate the credibility of above value, consider the equation $4, G$ is directly proportional to $X^{\prime}$. In the equation $5, X^{\prime}$ is directly proportional to $x$. Then, $G$ is in proportional to $x$, which is the shift distance of the shadow. In this experiment, measurement of $x$ is the key issue. In the process of determining $x$, we gain the proportion of uncertainty $\delta$ is $2.5 \%$. Thus, for the value of $G$, we think its uncertainty is about $2.5 \%$.

After we gain the first value of $G$, we do this experiment again. At the second time, we set the center distance of two balls to $0.42 \mathrm{~m}$, it is different from the first time. Other parameters are same. In the second experiment, the unanticipated result appears. We obtain the different value $G=6.785245 \times 10^{-11} \mathrm{~m}^{3} \mathrm{~kg}^{-1} \mathrm{~S}^{-2}$, with the uncertainty of about $2.3 \%$. We check the experimental process and data carefully, no any mistake is found. The second experimental value of $G$ is different from the first indeed.

In the next step, we compare our two values with other previous values.

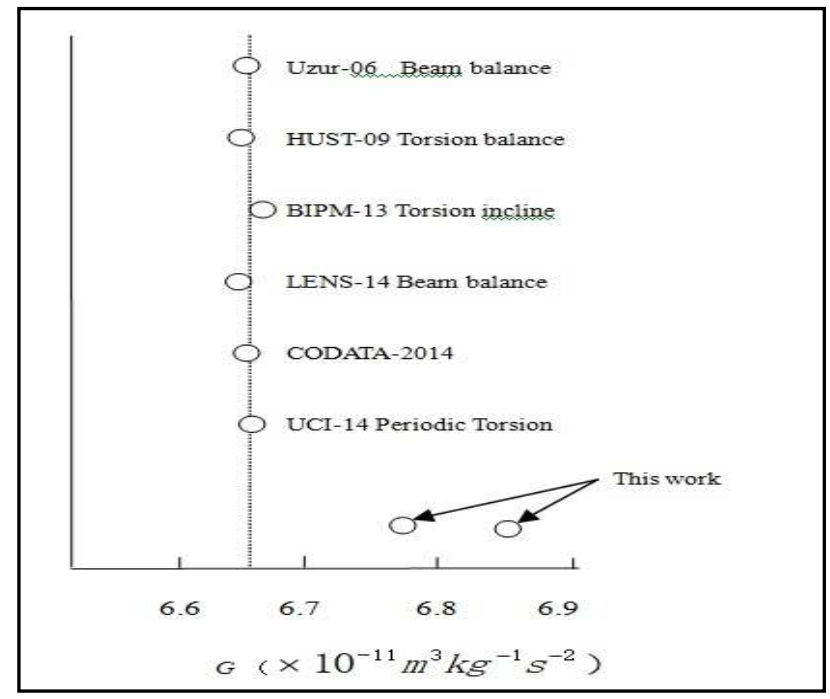

Figure 5: Comparison with previous results. (For the details of the abbreviations on the experiment identification, please see ref. Mohr et al., 2012 and Li et al., 2014) 


\section{QING-GUI: TWO VALUES OF THE CONSTANT G OBTAINED FROM INCLINING-PULL PROJECTION METHOD}

As shown in Figure 5, the values of our work are compared with that obtained in previous experiments and with 2014 CODATA adjustments. From the figure we can see that both of our values are larger than others. Perhaps, the reason lies in the conceptual difference of the experiment.

Here, we would like to make an analysis on the systematic error of torsion experiment. As shown in Figure 6 , when the big ball attracts the small ball on its own side, it should attract the other small one on the opposite side (Xue et al., 2014) (Rosi et al., 2014) (Newman et al., 2014) (Fan et al., 2008) (Quan et al., 2014). This can lead to the notable systematic error. What's more, this is the reason of why torsion experiment can only determine $G$ between small objects. To this day, this problem has not been solved. However, in our experiment, such systematic errors have been avoided. And our experiment can determine $G$ between big objects.

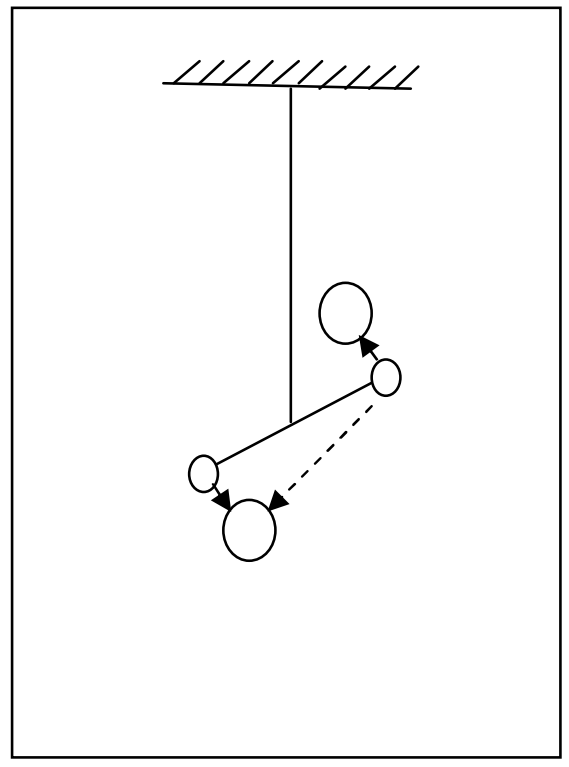

Figure 6: The big ball attract the two small balls in the torsion experiment

Measurements based on different methods are important for us to realize the gravitational constant $G$. For the source of the gravity, the author has proposed the conjecture that it is originated from the resultant force of the attraction and repulsion between the numberless particles in two macro objects. The gravity is the macroscopic performance of numberless micro-resultant forces. Maybe, its value has something indeed to do with the parameters such as object size, mass density and so on. It is possible for $G$ between celestial bodies not to equal with that between objects of several kilograms. Even if it is a constant on Earth, it does not mean it is a constant in the whole universe. This opinion had been mentioned in our other paper (Qing-gui, 2008).

\section{OUTLOOK AND CONCLUSION}

The gravitation between the objects is too small to detect easily. More than one hundred years after Newton put forward the theory of gravitation, Cavendish designed the torsion balance to determine value of $G$. This method is still in use today. In this paper, we report the new inclining-pull projection method to determine $G$. it is different from Cavendish's torsion experiment. Conceptually different experiments will help us to realize theory of gravitation farther. In the new experiment, we use big ball to attract the suspended small one, use projection to amplify the shift displacement so as to measure $G$ simply. Through the new experiment, we gain two different values $G=6.858448 \times 10^{-11} \mathrm{~m}^{3} \mathrm{~kg}^{-1} \mathrm{~S}^{-2}$, with uncertainty of about $2.5 \% ; G=6.785245 \times 10^{-11} \mathrm{~m}^{3} \mathrm{~kg}^{-1} \mathrm{~s}^{-2}$, with the uncertainty of about $2.3 \%$.

For the source of the gravity, we think, it is originated from the resultant force of the attraction and repulsion between the numberless particles in two macro objects. It is the macroscopic performance of numberless micro-resultant forces. Next step, we plan to improve the measurement accuracy by the following means: (a) extend the suspension line to about 100 meters, (b) Increase the weight of attracting object to $1000 \mathrm{~kg}$, (c) adopt the light source with smaller emitting area, (d) adopt the photometer with higher sensitivity. We believe that, in near future, the 


\section{QING-GUI: TWO VALUES OF THE CONSTANT G OBTAINED FROM INCLINING-PULL PROJECTION METHOD}

error will drop to lower than $1 \%$. This can help to solve the puzzle of $G$.

\section{ACKNOWLEDGMENT}

The author HU Qinggui acknowledges the financial support from The National Natural Science Foundation of China (Grant No.61275080).

\section{REFERENCES}

Cavendish H., 1798. Experiments to determine the density of the Earth. Phil. Trans. R. Soc. B., 88: 469-526.

Schlamminger S., 2014. Fundamental constants: a cool way to measure big G. Nature, 510: 478-480.

Gibney E., 2014. Rivals join forces to nail down Big G. Nature, 514, 150-151.

Mohr P.J., Newell D.B. and Taylor B.N., 2016. CODATA recommended values of the fundamental physical constants: 2014. Rev. Mod. Phys., 88: 035009.

Gillies G.T., 1987. The Newtonian gravitational constant: an index of measurements. Metrologia, 24: 1-56.

Quinn T., 2014. Don't stop the quest to measure Big G. Nature, 505: 455.

Luo J., Wang W.M., Hu Z.K. and Wang X.L., 2001. Precise determination of separation between spherical attracting masses in measuring the gravitational constant. Chin. Phys. Lett., 18: 1012-1014.

Rothleitner C. and Schlamminger S., 2017. Measurements of the Newtonian constant of gravitation, G. Rev. Sci. Instrum., 88: 111101.

Li Q. et al., 2016. Research on supporting mounts of spheres in measurement of gravitational constant G. Rev. Sci. Instrum., 87: 034504.

Gundlach J.H. and Merkowitz S.M., 2000. Measurement of Newton's constant using a torsion balance with angular acceleration feedback. Phys. Rev. Lett., 85: 2869-2872.
Liu L.X. et al., 2008. Measurement of density inhomogeneity for glass pendulum. Chin. Phys. Lett., 25: 4203-4206.

Newman R.D. and Bantel M.K., 1999. On determining G using a cryogenic torsion pendulum. Meas. Sci. Technol., 10: 445-453.

Qing-gui H., 2008. New experimental scheme to measure the gravitational constant $\mathrm{G}$ among big objects. Journal of Hebei University of Science and Technology, 29: 115 (in chinese).

Luo J., Hu Z.K., Fu X.H., Fan S.H. and Tang M.X., 1998. Determination of the Newtonian gravitational constant $\mathrm{G}$ with a nonlinear ftting method. Phys. Rev. D., 59: 042001.

Yang S.Q. et al., 2009. Direct measurement of the anelasticity of a tungsten fber. Phys. Rev. D., 80: 122005.

Griggs C.E., Moody M.V. and Norton R.S., 2017. Sensitive Superconducting Gravity Gradiometer Constructed with Levitated Test Masses. Physical Review Applied, 8: 064024.

Uyar K. and Ulker E., 2017. B-spline curve fitting with invasive weed optimization. Applied Mathematical Modelling, 52: 320.

Mohr P.J., Taylor B.N. and Newell D.B., 2012. CODATA recommended values of the fundamental physical constants: 2010. Rev. Mod. Phys., 84: 1527-1605.

Li Q. et al., 2014. G measurements with time-of-swing method at HUST. Phil. Trans. R. Soc. A, 372: 20140141.

Xue C. et al., 2014. Preliminary determination of Newtonian gravitational constant with angular acceleration feedback method. Phil. Trans. R. Soc. A 372: 20140031. 
QING-GUI: TWO VALUES OF THE CONSTANT $G$ OBTAINED FROM INCLINING-PULL PROJECTION METHOD

Rosi G., Sorrentino F., Cacciapuoti L., Prevedelli M. and Tino G.M., 2014. Precision measurement of the Newtonian gravitational constant using cold atoms. Nature, 510: 518-521.

Newman R., Bantel M., Berg E. and Cross W., 2014. A measurement of $\mathrm{G}$ with a cryogenic torsion pendulum. Phil. Trans. R. Soc. A 372: 20140025.
Fan X.D. et al., 2008. Coupled modes of the torsion pendulum. Phys. Lett. A 372: 547-552.

Quan L.D. et al., 2014. Feedback control of torsion balance in measurement of gravitational constant $\mathrm{G}$ with angular acceleration method. Rev. Sci. Instrum., 85: 014501. 